\title{
Lorentz Invariance Violation and Modified Hawking Fermions Tunneling Radiation
}

\author{
Shu-Zheng Yang, ${ }^{1}$ Kai Lin, ${ }^{2,3}$ Jin Li, $^{4}$ and Qing-Quan Jiang ${ }^{1}$ \\ ${ }^{1}$ Department of Astronomy, China West Normal University, Nanchong, Sichuan 637002, China \\ ${ }^{2}$ Instituto de Física e Química, Universidade Federal de Itajubá, 37500-903 Itajubá, MG, Brazil \\ ${ }^{3}$ Instituto de Física, Universidade de São Paulo, CP 66318, 05315-970 São Paulo, SP, Brazil \\ ${ }^{4}$ Department of Physics, Chongqing University, Chongqing 400030, China
}

Correspondence should be addressed to Kai Lin; 1k314159@hotmail.com

Received 20 April 2016; Accepted 8 June 2016

Academic Editor: Giulia Gubitosi

Copyright (C) 2016 Shu-Zheng Yang et al. This is an open access article distributed under the Creative Commons Attribution License, which permits unrestricted use, distribution, and reproduction in any medium, provided the original work is properly cited. The publication of this article was funded by SCOAP ${ }^{3}$.

Recently the modified Dirac equation with Lorentz invariance violation has been proposed, which would be helpful to resolve some issues in quantum gravity theory and high energy physics. In this paper, the modified Dirac equation has been generalized in curved spacetime, and then fermion tunneling of black holes is researched under this correctional Dirac field theory. We also use semiclassical approximation method to get correctional Hamilton-Jacobi equation, so that the correctional Hawking temperature and correctional black hole's entropy are derived.

\section{Introduction}

In 1974, Hawking proved black holes could radiate Hawking radiation once considering the quantum effect near the horizons of black holes $[1,2]$. This theory indicates that black hole would be viewed as thermodynamic system, so that black hole physics can be connected closely with gravity, quantum theory, and thermodynamics physics. According to the view point of quantum tunneling theory, the virtual particles inside of black hole could cross the horizon due to the quantum tunneling effect and become real particles and then could be observed by observers as Hawking radiation. Wilczek et al. proposed a semiclassical method to study the quantum tunneling from the horizon of black hole [321]. Along with this method, the Hamilton-Jacobi method was applied to calculate the Hawking tunneling radiation. According to the Hamilton-Jacobi method, the wave function of Klein-Gordon equation can be rewritten as $\Phi=$ $C \exp (i S / \hbar)$ (where $S$ is semiclassical action) and HamiltonJacobi equation is obtained via semiclassical approximation. Using the Hamilton-Jacobi equation, the tunneling rate could be calculated by the relationship $\Gamma \sim \exp (-2 \operatorname{Im} S$ ) (where $\Gamma$ is the tunneling rate at the horizon of black hole), and then the Hawking temperature can be determined. People have applied this method to research Hawking tunneling radiation of several static, stationary, and dynamical black holes.

However, since the Hamilton-Jacobi equation is derived from Klein-Gordon equation, original Hamilton-Jacobi method just can be valid for scalar particles in principle. Therefore, Kerner and Mann studied fermions tunneling of black hole by a new method [22-32], which assumes the wave function of Dirac equation $\Psi$ as spin-up and spin-down and then calculates the fermions tunneling, respectively. Nevertheless, this method is still impossible to apply in arbitrary dimensional spacetime. Our work in 2009 showed that the Hamilton-Jacobi equation can also be derived from Dirac equation via semiclassical approximation, so we proved that the Hamilton-Jacobi equation can be used to study the fermions tunneling directly [33-35].

On the other hand, as the basis of general relativity and quantum field theory, Lorentz invariance is proposed to be spontaneously violated at higher energy scales. A possible deformed dispersion relation is given by [36-44]

$$
p_{0}^{2}=\vec{p}^{2}+m^{2}-\left(L p_{0}\right)^{\alpha} \vec{p}^{2}
$$


where $p_{0}$ and $\vec{p}$ are the energy and momentum of particle and $L$ is "minimal length" with the order of the Plank length. The work of spacetime foam Liouville string models has introduced this relation with $\alpha=1$, and people also proposed quantum equation of spineless particles by using this relation. Recently, Kruglov considers the deformed dispersion relation with $\alpha=2$ and proposes modified Dirac equation [45]:

$$
\left[\bar{\gamma}^{\mu} \partial_{\mu}+m-i L\left(\bar{\gamma}^{t} \partial_{t}\right)\left(\bar{\gamma}^{j} \partial_{j}\right)\right] \psi=0
$$

where $\bar{\gamma}^{a}$ is ordinary gamma matrix and $j$ is space coordinate, while $\mu$ is spacetime coordinate. The effect of the correctional term would be observed in higher energy experiment.

In this paper, we try to generalize the modified Dirac equation in curved spacetime and then study the correction of Hawking tunneling radiation. In Section 2, the modified Dirac equation in curved spacetime is constructed and then the modified Hamilton-Jacobi equation is derived via semiclassical approximation. We apply the modified HamiltonJacobi equation to the fermions tunneling radiation of $2+$ 1-dimensional black string and higher dimensional BTZlike black strings in Sections 3 and 4, respectively, and Section 5 includes some conclusion and the discussion about the correction of black hole's entropy.

\section{Modified Dirac Equation and Hamilton-Jacobi Equation in Curved Spacetime}

As we all know, the gamma matrix and partial derivative should become gamma matrix in curved spacetime $\gamma^{a}$ and covariant $\mathscr{D}_{a}$ derivative, respectively, namely,

$$
\begin{aligned}
& \bar{\gamma}^{a} \longrightarrow \gamma^{a} \\
& \partial_{a} \longrightarrow \mathscr{D}_{a}=\partial_{a}+\Omega_{a}+\frac{i}{\hbar} e A_{a}
\end{aligned}
$$

where $\gamma^{a}$ satisfy the relationship $\left\{\gamma^{a}, \gamma^{b}\right\}=\gamma^{a} \gamma^{b}+\gamma^{b} \gamma^{a}=$ $2 g^{a b} I, e A_{a}$ is charged term of Dirac equation, and $\Omega_{\mu}=$ $(1 / 8)\left(\gamma^{a} \gamma^{b}-\gamma^{b} \gamma^{a}\right) e_{a}^{v}\left(\partial_{\mu} e_{b \nu}-\Gamma_{\mu \nu}^{c} e_{b c}\right)$ is spin connection. According to this transformation, we can construct the modified Dirac equation in curved spacetime as

$$
\left[\gamma^{\mu} \mathscr{D}_{\mu}+\frac{m}{\hbar}-\sigma \hbar\left(\gamma^{t} \mathscr{D}^{t}\right)\left(\gamma^{j} \mathscr{D}^{j}\right)\right] \Psi=0
$$

where we choose $c=1$ but $\hbar \neq 1$, while $c=\hbar=1$ in (1) and (2). It is assumed that $\sigma \ll 1$, so that the correctional term $\sigma \hbar\left(\gamma^{t} \mathscr{D}^{t}\right)\left(\gamma^{j} \mathscr{D}^{j}\right)$ is very small.

Now let us use the modified Dirac equation to derive the modified Hamilton-Jacobi equation. Firstly, we rewrite the wave function of Dirac equation as [33-35]

$$
\Psi=\zeta\left(t, x^{j}\right) \exp \left[\frac{i}{\hbar} S\left(t, x^{j}\right)\right]
$$

where $\zeta\left(t, x^{j}\right)$ and $\Psi$ are $m \times 1$ matrices and $\partial_{t} S=-\omega$. In semiclassical approximation, we can consider that $\hbar$ is very small, so that we can neglect the terms with $\hbar$ after dividing by the exponential terms and multiplying by $\hbar$. Therefore, (4) is rewritten as

$$
\begin{aligned}
& {\left[i \gamma^{\mu}\left(\partial_{\mu} S+e A_{\mu}\right)+m-\sigma \gamma^{t}\left(\omega-e A_{t}\right) \gamma^{j}\left(\partial_{j} S+e A_{j}\right)\right]} \\
& \cdot \zeta\left(t, x^{j}\right)=0 .
\end{aligned}
$$

Considering the relationship

$$
\gamma^{\mu}\left(\partial_{\mu} S+e A_{\mu}\right)=-\gamma^{t}\left(\omega-e A_{t}\right)+\gamma^{j}\left(\partial_{j} S+e A_{j}\right),
$$

we can get

$$
\left[i \Gamma^{\mu}\left(\partial_{\mu} S+e A_{\mu}\right)+M\right] \zeta\left(t, x^{j}\right)=0,
$$

where

$$
\begin{aligned}
& \Gamma^{\mu}=\left[1+i \sigma\left(\omega-e A_{t}\right) \gamma^{t}\right] \gamma^{\mu}, \\
& M=m-\sigma g^{t t}\left(\omega-e A_{t}\right)^{2} .
\end{aligned}
$$

Now, multiplying both sides of (9) by the matrix $-i \Gamma^{\nu}\left(\partial_{\gamma} S+\right.$ $e A_{\gamma}$ ), we can obtain

$$
\begin{array}{r}
\Gamma^{\nu}\left(\partial_{\nu} S+e A_{\nu}\right) \Gamma^{\mu}\left(\partial_{\mu} S+e A_{\mu}\right) \zeta \\
-i M \Gamma^{\nu}\left(\partial_{\nu} S+e A_{\nu}\right) \zeta=0 .
\end{array}
$$

The second term of the above equation could be simplified again by (8), so the above equation can be rewritten as

$$
\Gamma^{\nu} \Gamma^{\mu}\left(\partial_{\nu} S+e A_{\nu}\right)\left(\partial_{\mu} S+e A_{\mu}\right) \zeta+M^{2} \zeta=0,
$$

where we can prove the relation

$$
\Gamma^{\nu} \Gamma^{\mu}=\gamma^{\nu} \gamma^{\mu}+2 i \sigma\left(\omega-e A_{t}\right) g^{t v} \gamma^{\mu}+\mathcal{O}\left(\sigma^{2}\right) .
$$

We always ignore $\mathcal{O}\left(\sigma^{2}\right)$ terms because $\sigma$ is very small. Now, let us exchange the position of $\mu$ and $\nu$ in (11) and consider the relation of gamma matrices $\left\{\gamma^{a}, \gamma^{b}\right\}=2 g^{a b} I$; then we can obtain

$$
\begin{aligned}
& \left\{\frac{\gamma^{\alpha} \gamma^{\beta}+\gamma^{\alpha} \gamma^{\beta}}{2}\left(\partial_{\alpha} S+e A_{\alpha}\right)\left(\partial_{\beta} S+e A_{\beta}\right)+m^{2}\right. \\
& \quad-2 \sigma m g^{t t}\left(\omega-e A_{t}\right)^{2} \\
& \left.+2 i \sigma\left(\omega-e A_{t}\right) g^{t \rho}\left(\partial_{\rho} S+e A_{\rho}\right) \gamma^{\mu}\left(\partial_{\mu} S+e A_{\mu}\right)\right\} \\
& +\zeta+\mathcal{O}\left(\sigma^{2}\right)=\left\{g^{\alpha \beta}\left(\partial_{\alpha} S+e A_{\alpha}\right)\left(\partial_{\beta} S+e A_{\beta}\right)\right. \\
& +m^{2}-2 \sigma m g^{t t}\left(\omega-e A_{t}\right)^{2} \\
& \left.+2 i \sigma\left(\omega-e A_{t}\right) g^{t \rho}\left(\partial_{\rho} S+e A_{\rho}\right) \gamma^{\mu}\left(\partial_{\mu} S+e A_{\mu}\right)\right\} \zeta \\
& +\mathcal{O}\left(\sigma^{2}\right)=0 .
\end{aligned}
$$

Namely,

$$
\left[i \sigma \gamma^{\mu}\left(\partial_{\mu} S+e A_{\mu}\right)+\mathscr{M}\right] \zeta\left(t, x^{j}\right)=0
$$


where

$$
=\frac{g^{\alpha \beta}\left(\partial_{\alpha} S+e A_{\alpha}\right)\left(\partial_{\beta} S+e A_{\beta}\right)+m^{2}-2 \sigma m g^{t t}\left(\omega-e A_{t}\right)^{2}}{2 g^{t \rho}\left(\partial_{\rho} S+e A_{\rho}\right)\left(\omega-e A_{t}\right)} .
$$

Using the idea of (10)-(11) again, we can multiply both sides of (15) by the matrix $-i \gamma^{\nu}\left(\partial_{\gamma} S+e A_{\nu}\right)$, so that the equation becomes

$$
\begin{gathered}
\sigma \gamma^{\nu}\left(\partial_{\nu} S+e A_{\nu}\right) \gamma^{\mu}\left(\partial_{\mu} S+e A_{\mu}\right) \zeta \\
-i \mathscr{M} \gamma^{\nu}\left(\partial_{\nu} S+e A_{\nu}\right) \zeta=0 .
\end{gathered}
$$

The second term of the above equation could be simplified again by (15). Then, exchange $\mu$ and $\nu$ and use the relationship $\left\{\gamma^{a}, \gamma^{b}\right\}=2 g^{a b} I$, so the above equation can be rewritten as

$$
\begin{aligned}
& {\left[\frac{\gamma^{\nu} \gamma^{\mu}+\gamma^{\mu} \gamma^{\nu}}{2} \sigma^{2}\left(\partial_{\nu} S+e A_{\nu}\right)\left(\partial_{\mu} S+e A_{\mu}\right)+\mathscr{M}^{2}\right]} \\
& \cdot \zeta\left(t, x^{j}\right) \\
& \quad=\left[\sigma^{2} g^{\mu \nu}\left(\partial_{\nu} S+e A_{\nu}\right)\left(\partial_{\mu} S+e A_{\mu}\right)+\mathscr{M}^{2}\right] \zeta\left(t, x^{j}\right) \\
& \quad=0 .
\end{aligned}
$$

The condition that (17) has nontrivial solution required the determinant of coefficient in (17) should vanish, so we can directly get the equation

$$
\sigma^{2} g^{\mu \nu}\left(\partial_{\gamma} S+e A_{\nu}\right)\left(\partial_{\mu} S+e A_{\mu}\right)+\mathscr{M}^{2}=0 .
$$

Consider the square root for left side of (18) and ignore all $\mathcal{O}\left(\sigma^{2}\right)$ terms, so we can directly get the modified HamiltonJacobi equation:

$$
\begin{gathered}
g^{\mu \nu}\left(\partial_{\nu} S+e A_{\nu}\right)\left(\partial_{\mu} S+e A_{\mu}\right)+m^{2} \\
-2 \sigma m g^{t t}\left(\omega-e A_{t}\right)^{2}=0 .
\end{gathered}
$$

Therefore, we find that the modified Dirac equation from Lorentz invariance violation could lead to the modified Hamilton-Jacobi equation, and the correction of HamiltonJacobi equation depends on the energy and mass of radiation fermions. Using the modified Hamilton-Jacobi equation, we then investigate the fermions Hawking tunneling radiation of $2+1$-dimensional black string and $n+1$-dimensional BTZlike string in the following two sections.

\section{Fermions Tunneling of $2+1$-Dimensional Black String}

The research of gravity in $2+1$ dimension can help people further understand the properties of gravity, and it is also important to construct the quantum gravity. Recently, Murata et al. have researched the $2+1$-dimensional gravity with dilaton field, whose action is given by [46]

$$
I=M_{3} \int d^{3} x \sqrt{-g}\left(B R+\frac{\lambda^{2}}{B}\right),
$$

where $B, M_{3}$, and $\lambda$ are, respectively, the dilaton field, 3dimensional Planck mass, and the parameter with mass dimension. The static black string solution is given by

$$
\begin{aligned}
d s^{2} & =-\ln \left(\frac{r}{r_{H}}\right) d t^{2}+\ln \left(\frac{r}{r_{H}}\right)^{-1} d r^{2}+d y^{2}, \\
B & =\lambda r .
\end{aligned}
$$

It is evident that the horizon of this black hole is $r_{H}$, but the black string is unstable as $r_{H} \lesssim \mathscr{L}$ (where $\mathscr{L}$ is scale of compactification), so it is assumed that $r_{H} \gg \mathscr{L}$.

Now we research the fermions tunneling of this black hole, so the modified Hamilton-Jacobi equation in this spacetime is given by

$$
\begin{gathered}
-(1-2 \sigma m) \ln \left(\frac{r}{r_{H}}\right)^{-1} \omega^{2}+\ln \left(\frac{r}{r_{H}}\right)\left(\frac{d R}{d r}\right)^{2} \\
+\left(\frac{d Y}{d y}\right)^{2}+m^{2}=0,
\end{gathered}
$$

where we have set $S=-\omega t+R(r)+Y(y)$, yielding the radial Hamilton-Jacobi equation as

$$
\begin{aligned}
& -(1-2 \sigma m) \ln \left(\frac{r}{r_{H}}\right)^{-1} \omega^{2}+\ln \left(\frac{r}{r_{H}}\right)\left(\frac{d R}{d r}\right)^{2}+\lambda_{0} \\
& +m^{2}=0
\end{aligned}
$$

where the constant $\lambda_{0}$ is from separation of variables, and (23) finally can be written as

$$
\begin{aligned}
R_{ \pm}(r)= & \pm \int \ln \left(\frac{r}{r_{H}}\right)^{-1} \\
& \cdot \sqrt{(1-2 \sigma m) \omega^{2}-\ln \left(\frac{r}{r_{H}}\right)\left(\lambda_{0}+m^{2}\right)} .
\end{aligned}
$$

At the horizon $r_{H}$ of the black string, the above equation is integrated via residue theorem, and we can get

$$
R_{ \pm}(r)= \pm i \pi(1-\sigma m) r_{H} \omega
$$

and the fermions tunneling rate

$$
\begin{aligned}
\Gamma & =\exp \left(-\frac{2}{\hbar} \operatorname{Im} S\right)=\exp \left[-\frac{2}{\hbar}\left(\operatorname{Im} R_{+}-\operatorname{Im} R_{-}\right)\right] \\
& =e^{-(4 \pi / \hbar)(1-\sigma m) r_{H} \omega}=e^{-\omega / T_{H}} .
\end{aligned}
$$

The relationship between tunneling rate and Hawking temperature required

$$
T_{H}=\hbar \frac{1+\sigma m}{4 \pi r_{H}}=(1+\sigma m) T_{0},
$$

where $T_{H}$ and $T_{0}$ are the modified and nonmodified Hawking temperature of the $2+1$-dimensional black string, respectively, and $\sigma$ term is the correction. 


\section{Fermions Tunneling of Higher Dimensional BTZ-Like Black Strings}

As we all know that the linear Maxwell action fails to satisfy the conformal symmetry in higher dimensional spacetime $[47,48]$, so Hassaïne and Martínez proposed gravity theory with nonlinear Maxwell field in arbitrary dimensional spacetime:

$$
I=-\frac{1}{16 \pi} \int_{M} d^{n+1} x \sqrt{-g}\left[R+\frac{2}{l^{2}}-\beta\left(\alpha F_{\mu \nu} F^{\mu \nu}\right)^{s}\right],
$$

where $\Lambda \equiv-l^{-2}$ is cosmological constant. Hendi researched $n+1$-dimensional static black strings solution with $\beta=1$, $\alpha=-1$, and $s=n / 2$, and it is charged BTZ-like solutions [49], whose metric is

$$
d s^{2}=-f(r) d t^{2}+\frac{d r^{2}}{f(r)}+r^{2} \sum_{k}\left(d x^{k}\right)^{2}
$$

where

$$
f(r)=\frac{r^{2}}{l^{2}}-r^{2-n}\left(M+2^{n / 2} Q^{n-1} A_{t}\right),
$$

and the electromagnetic potential is

$$
A=A_{t} d t=Q \ln \left(\frac{r}{l}\right) d t
$$

As $n=2$, this solution is no other than the static charged BTZ solution. We will study the Hawking radiation and black hole temperature at the event horizon $r_{H}$ of this black string. In (19), we can set $S=-\omega t+R(r)+Y\left(x^{k}\right)$, where $x^{k}$ are the space coordinates excluding the radial coordinate, so that the modified Hamilton-Jacobi equation is given by

$$
\begin{gathered}
-(1-2 \sigma m) f^{-1}(r)\left(\omega-e A_{t}\right)^{2}+f(r)\left(\frac{d R}{d r}\right)^{2} \\
+\frac{1}{r^{2}} \sum_{k}\left(\frac{d Y}{d x^{k}}\right)^{2}+m^{2}=0,
\end{gathered}
$$

and the radial equation with constant $\lambda_{0}$ is

$$
\begin{aligned}
& -(1-2 \sigma m) f^{-1}(r)\left(\omega-e A_{t}\right)^{2}+f(r)\left(\frac{d R}{d r}\right)^{2}+\frac{\lambda_{0}}{r^{2}} \\
& +m^{2}=0 .
\end{aligned}
$$

Therefore, at the horizon $r_{H}$ of the black string, $f\left(r_{H}\right)=0$ and we finally get

$$
\begin{aligned}
& R_{ \pm}(r)= \pm \int f(r)^{-1} \\
& \cdot \sqrt{(1-2 \sigma m)\left(\omega-e A_{t}\right)^{2}-f(r)\left(\lambda_{0}+m^{2}\right)} \\
& = \pm i \pi(1-\sigma m) \frac{\omega-\omega_{0}}{f^{\prime}\left(r_{H}\right)}
\end{aligned}
$$

where $\omega_{0}=e A_{t}\left(r_{H}\right)$. This means that the fermions tunneling rate is

$$
\begin{aligned}
\Gamma & =\exp \left(-\frac{2}{\hbar} \operatorname{Im} S\right)=\exp \left[-\frac{2}{\hbar}\left(\operatorname{Im} R_{+}-\operatorname{Im} R_{-}\right)\right] \\
& =e^{-(4 \pi / \hbar)(1-\sigma m)\left(\left(\omega-\omega_{0}\right) / f^{\prime}\left(r_{H}\right)\right)}=e^{-\left(\left(\omega-\omega_{0}\right) / T_{H}\right)}
\end{aligned}
$$

and the Hawking temperature is

$$
\begin{aligned}
T_{H} & =\hbar \frac{1+\sigma m}{4 \pi} f^{\prime}\left(r_{H}\right) \\
& =\hbar \frac{1+\sigma m}{4 \pi}\left(\frac{n r_{H}}{l^{2}}-2^{n / 2} Q^{n} r_{H}^{1-n}\right)=(1+\sigma m) T_{0},
\end{aligned}
$$

where $T_{H}$ and $T_{0}$ are the modified and nonmodified Hawking temperature of the $n+1$-dimensional BTZ-like black string, respectively, and $\sigma$ term is the correction.

\section{Conclusions}

In this paper, we consider the deformed dispersion relation with Lorentz invariance violation and generalize the modified Dirac equation in curved spacetime. The fermions tunneling radiation of black strings is researched, and we find that the modified Dirac equation could lead to Hawking temperature's correction, which depends on the correction parameter $\sigma$ and particle mass $m$ in the modified Dirac equation. Next we will discuss the correction of black hole entropy in this theory.

The first law of black hole thermodynamics requires

$$
d M=T d S+\Xi d J+U d Q
$$

where $\Xi$ and $U$ are electromagnetic potential and rotating potential, so the nonmodified entropy of black hole is $[1,2$, $50,51]$

$$
d S_{0}=\frac{d M-\Xi d J-U d Q}{T_{0}} .
$$

From the above results, we know that the relationship between modified and nonmodified Hawking temperature is $T_{H}=(1+\sigma m) T_{0}$, since the nonmodified black hole entropy is given by

$$
\begin{aligned}
S_{H} & =\int d S_{H}=\int \frac{d M-\Xi d J-U d Q}{(1+\sigma m) T_{0}} \\
& =S_{0}-m \int \sigma d S_{0}+\mathcal{O}\left(\sigma^{2}\right),
\end{aligned}
$$

where we can ignore $\mathcal{O}\left(\sigma^{2}\right)$ because $\sigma \ll 1$. Equation (39) shows that the correction of black hole entropy depends on $\sigma$, which is independent from time and space coordinates. However, it is possible that $\sigma$ depends on other parameters in curved spacetime, and it is very interesting that $\sigma$ depends on $S_{0}$. In particular, as $\sigma=\sigma_{0} / S_{0}+\cdots$, we can get the logarithmic correction of black hole entropy

$$
S_{H}=S_{0}-m \sigma_{0} \ln S_{0}+\cdots \text {. }
$$


In quantum gravity theory, the logarithmic correction has been researched in detail [52-69], and according to [70], it is required that the coefficient of logarithmic correction should be $-(n+1) / 2(n-1)$ in $n+1$-dimensional spacetime, so it indicates that $\sigma_{0}$ could be $(n+1) / 2 m(n-1)$.

On the other hand, from the deformed dispersion relation (1) with $\alpha=2$, it is implied that the Klein-Gordon equation could be given by

$$
\left(-\partial_{t}^{2}+\partial_{j}^{2}+m^{2}-\sigma^{2} \hbar^{2} \partial_{t}^{2} \partial_{j}^{2}\right) \Phi=0
$$

so the generalized uncharged Klein-Gordon equation in static curved spacetime is

$$
\left[g^{t t} \nabla_{t}^{2}+g^{j j} \nabla_{j}^{2}+m^{2}+\sigma^{2} \hbar^{2}\left(g^{t t} \nabla_{t}^{2}\right)\left(g^{j j} \nabla_{j}^{2}\right)\right] \Phi=0,
$$

and using the semiclassical approximation with $\Phi=$ $C \exp (i S / \hbar)$, the modified Hamilton-Jacobi equation in scalar field is given by

$$
\left(1+\sigma^{2} g^{t t} \omega^{2}\right) g^{\mu \nu} \partial_{\mu} S \partial_{\nu} S+m^{2}-\sigma^{2}\left(g^{t t}\right)^{2} \omega^{4}=0 .
$$

Namely,

$$
\begin{aligned}
& g^{\mu \nu} \partial_{\mu} S \partial_{\nu} S+m^{2}-\sigma^{2} g^{t t} \omega^{2}\left(m^{2}+g^{t t} \omega^{2}\right)+\mathcal{O}\left(\sigma^{4}\right) \\
& \quad=0
\end{aligned}
$$

Contrasting (19) and (44) as uncharged case, we find that the correctional terms of Dirac field and scalar field are very different. The fact implies that the corrections of Hawking temperature and black hole entropy from Hawking tunneling radiation with different spin particles could be different, and this conclusion could be helpful to suggest a new idea to research the black hole information paradox. Work in these fields is currently in progress.

\section{Competing Interests}

The authors declare that they have no competing interests.

\section{Acknowledgments}

This work was supported by FAPESP no. 2012/08934-0, CNPq, CAPES, and National Natural Science Foundation of China no. 11573022, no. 11205254, no. 11178018, and no. 11375279 .

\section{References}

[1] S. W. Hawking, "Black hole explosions?"” Nature, vol. 248, no. 5443, pp. 30-31, 1974.

[2] S. W. Hawking, "Particle creation by black holes," Communications in Mathematical Physics, vol. 43, no. 3, pp. 199-220, 1975.

[3] S. P. Robinson and F. Wilczek, "Relationship between Hawking radiation and gravitational anomalies," Physical Review Letters, vol. 95, no. 1, Article ID 011303, 4 pages, 2005.

[4] T. Damoar and R. Ruffini, "Black-hole evaporation in the KleinSauter-Heisenberg-Euler formalism," Physical Review D, vol. 14, no. 2, p. 332, 1976.
[5] S. Sannan, "Heuristic derivation of the probability distributions of particles emitted by a black hole," General Relativity and Gravitation, vol. 20, no. 3, pp. 239-246, 1988.

[6] P. Kraus and F. Wilczek, "Self-interaction correction to black hole radiance," Nuclear Physics B, vol. 433, no. 2, pp. 403-420, 1995.

[7] M. K. Parikh and F. Wilczek, "Hawking radiation as tunneling," Physical Review Letters, vol. 85, article 5042, 2000.

[8] S. Hemming and E. Keski-Vakkuri, "Hawking radiation from AdS black holes," Physical Review D, vol. 64, no. 4, Article ID 044006, 8 pages, 2001.

[9] Q.-Q. Jiang, S.-Q. Wu, and X. Cai, "Hawking radiation from dilatonic black holes via anomalies," Physical Review D, vol. 75, Article ID 064029, 2007.

[10] S. Iso, H. Umetsu, and F. Wilczek, "Anomalies, Hawking radiations, and regularity in rotating black holes," Physical Review D, vol. 74, no. 4, Article ID 044017, 10 pages, 2006.

[11] A. J. Medved, "Radiation via tunneling from a de Sitter cosmological horizon," Physical Review D, vol. 66, Article ID 124009, 2002.

[12] M. K. Parikh, "Energy conservation and hawking radiation," in Proceedings of the 10th Marcel Grossmann Meeting, pp. 15851590, February 2006.

[13] M. K. Parikh, J. Y. Zhang, and Z. Zhao, "Hawking radiation of charged particles via tunneling from the Reissner-Nordström black hole," Journal of High Energy Physics, vol. 2005, no. 10, article 055, 2005.

[14] J. Zhang and Z. Zhao, "Charged particles' tunnelling from the Kerr-Newman black hole," Physics Letters B, vol. 638, no. 2-3, pp. 110-113, 2006.

[15] E. T. Akhmedov, V. Akhmedova, and D. Singleton, "Hawking temperature in the tunneling picture," Physics Letters B, vol. 642, no. 1-2, pp. 124-128, 2006.

[16] V. Akhmedova, T. Pilling, A. de Gill, and D. Singleton, “Temporal contribution to gravitational WKB-like calculations," Physics Letters B, vol. 666, no. 3, pp. 269-271, 2008.

[17] K. Srinivasan and T. Padmanabhan, "Particle production and complex path analysis," Physical Review D, vol. 60, no. 2, Article ID 024007, 20 pages, 1999.

[18] S. Shankaranarayanan, T. Padmanabhan, and K. Srinivasan, "Hawking radiation in different coordinate settings: complex paths approach," Classical and Quantum Gravity, vol. 19, no. 10, pp. 2671-2687, 2002.

[19] M. Angheben, M. Nadalini, L. Vanzo, and S. Zerbini, "Hawking radiation as tunneling for extremal and rotating black holes," Journal of High Energy Physics, vol. 2005, no. 5, article 014, 18 pages, 2005.

[20] S. Z. Yang and D. Y. Chen, "Hawking radiation as tunneling from the vaidya-bonner black hole," International Journal of Theoretical Physics, vol. 46, no. 11, pp. 2923-2927, 2007.

[21] Y. Shu-Zheng and C. De-You, "Tunnelling effect of the nonstationary Kerr black hole," Chinese Physics B, vol. 17, no. 3, pp. 817-821, 2008.

[22] R. Kerner and R. B. Mann, "Fermions tunnelling from black holes," Classical and Quantum Gravity, vol. 25, no. 9, Article ID 095014, 2008.

[23] R. Kerner and R. B. Mann, "Charged fermions tunnelling from kerr-newman black holes," Physics Letters B, vol. 665, no. 4, pp. 277-283, 2008.

[24] R. Li, J.-R. Ren, and S.-W. Wei, "Hawking radiation of Dirac particles via tunneling from the Kerr black hole," Classical and 
Quantum Gravity, vol. 25, no. 12, Article ID 125016, 5 pages, 2008.

[25] R. Li and J.-R. Ren, "Dirac particles tunneling from BTZ black hole," Physics Letters B, vol. 661, no. 5, pp. 370-372, 2008.

[26] D.-Y. Chen, Q.-Q. Jiang, and X.-T. Zu, "Fermions tunnelling from the charged dilatonic black holes," Classical and Quantum Gravity, vol. 25, no. 20, Article ID 205022, 2008.

[27] D.-Y. Chen, Q.-Q. Jiang, and X.-T. Zu, "Hawking radiation of Dirac particles via tunnelling from rotating black holes in de Sitter spaces," Physics Letters B, vol. 665, no. 2-3, pp. 106-110, 2008.

[28] R. Di Criscienzo and L. Vanzo, "Fermion tunneling from dynamical horizons," Europhysics Letters, vol. 82, no. 6, Article ID 60001, 2008.

[29] H. Li, S. Yang, T. Zhou, and R. Lin, "Fermion tunneling from a Vaidya black hole," Europhysics Letters, vol. 84, no. 2, Article ID 20003, 2008.

[30] Q.-Q. Jiang, "Fermions tunnelling from GHS and non-extremal D1-D5 black holes," Physics Letters B, vol. 666, no. 5, pp. 517-521, 2008.

[31] Q.-Q. Jiang, "Dirac particle tunneling from black rings," Physical Review D, vol. 78, Article ID 044009, 2008.

[32] K. Lin and S. Z. Yang, "Quantum tunneling from apparent horizon of rainbow-FRW universe," International Journal of Theoretical Physics, vol. 48, no. 7, pp. 2061-2067, 2009.

[33] Y. Zhou, "D4 brane probes in gauge/gravity duality," Physical Review D, vol. 79, Article ID 066005, 2009.

[34] K. Lin and S. Yang, "Fermions tunneling of higher-dimensional Kerr-anti-de Sitter black hole with one rotational parameter," Physics Letters B, vol. 674, no. 2, pp. 127-130, 2009.

[35] K. Lin and S. Z. Yang, "A simpler method for researching fermions tunneling from black holes," Chinese Physics B, vol. 20, no. 11, Article ID 110403, 2011.

[36] G. Amelino-Camelia, "Relativity in spacetimes with shortdistance structure governed by an observer-independent (Planckian) length scale," International Journal of Modern Physics D: Gravitation, Astrophysics, Cosmology, vol. 11, article 35, 2002.

[37] G. Amelino-Camelia, "Phenomenology of Planck-scale Lorentz-symmetry test theories," New Journal of Physics, vol. 6, p. $188,2004$.

[38] J. Magueijo and L. Smolin, "Lorentz invariance with an invariant energy scale," Physical Review Letters, vol. 88, no. 19, Article ID 0112090, 2002.

[39] J. Magueijo and L. Smolin, "Generalized Lorentz invariance with an invariant energy scale," Physical Review D, vol. 67, no. 4, Article ID 044017, 2003.

[40] J. Ellis, N. E. Mavromatos, and D. V. Nanopoulos, "String theory modifies quantum mechanics," Physics Letters B, vol. 293, no. 12, pp. 37-48, 1992.

[41] J. Ellis, N. E. Mavromatos, and D. V. Nanopoulos, "A microscopic Liouville arrow of time," Chaos, Solitons \& Fractals, vol. 10, no. 2-3, pp. 345-363, 1999.

[42] J. R. Ellis, N. E. Mavromatos, and A. S. Sakharov, "Synchrotron radiation from the Crab Nebula discriminates between models of space-time foam," Astroparticle Physics, vol. 20, no. 6, pp. 669-682, 2004.

[43] S. I. Kruglov, "Modified wave equation for spinless particles and its solutions in an external magnetic field," Modern Physics Letters A, vol. 28, Article ID 1350014, 2013.
[44] T. Jacobson, S. Liberati, and D. Mattingly, "A strong astrophysical constraint on the violation of special relativity by quantum gravity," Nature, vol. 424, pp. 1019-1021, 2003.

[45] S. I. Kruglov, "Modified Dirac equation with Lorentz invariance violation and its solutions for particles in an external magnetic field," Physics Letters B, vol. 718, no. 1, pp. 228-231, 2012.

[46] K. Murata, J. Soda, and S. Kanno, "Evaporating (2+1)dimensional black strings," http://arxiv.org/abs/gr-qc/0701137.

[47] M. Hassaïne and C. Martínez, "Higher-dimensional black holes with a conformally invariant Maxwell source," Physical Review $D$, vol. 75, Article ID 027502, 2007.

[48] M. Hassaïne and C. Martínez, "Higher-dimensional charged black hole solutions with a nonlinear electrodynamics source," Classical and Quantum Gravity, vol. 25, no. 19, 2008.

[49] S. H. Hendi, "Charged BTZ-like black holes in higher dimensions," The European Physical Journal C, vol. 71, article 1551, 2007.

[50] J. D. Bekenstein, "Black holes and the second law," Lettere al Nuovo Cimento, vol. 4, no. 15, pp. 737-740, 1972.

[51] J. D. Bekenstein, "Black holes and entropy," Physical Review D. Particles and Fields. Third Series, vol. 7, pp. 2333-2346, 1973.

[52] R. Banerjee and B. R. Majhi, "Quantum tunneling beyond semiclassical approximation," Journal of High Energy Physics, vol. 2008, no. 6, article 095, 20 pages, 2008.

[53] R. Banerjee and B. R. Majhi, "Quantum tunneling and back reaction," Physics Letters B, vol. 662, no. 1, pp. 62-65, 2008.

[54] R. Banerjee, B. R. Majhi, and S. Samanta, "Noncommutative black hole thermodynamics," Physical Review D, vol. 77, Article ID 124035, 2008.

[55] R. Banerjee and R. B. Majhi, "Connecting anomaly and tunneling methods for the Hawking effect through chirality," Physical Review D, vol. 79, Article ID 064024, 2009.

[56] S. K. Modak, "Corrected entropy of BTZ black hole in tunneling approach," Physics Letters B, vol. 671, no. 1, pp. 167-173, 2009.

[57] R. Banerjee and B. R. Majhi, "Quantum tunneling and trace anomaly," Physics Letters B, vol. 674, no. 3, pp. 218-222, 2009.

[58] R.-G. Cai, L.-M. Cao, and Y.-P. Hu, "Corrected entropy-area relation and modified Friedmann equations," Journal of High Energy Physics, vol. 2008, no. 8, article 090, 12 pages, 2008.

[59] J. Y. Zhang, "Black hole quantum tunnelling and black hole entropy correction," Physics Letters B, vol. 668, no. 5, pp. 353356, 2008.

[60] R. Banerjee and S. K. Modak, "Exact differential and corrected area law for stationary black holes in tunneling method," Journal of High Energy Physics, vol. 2009, no. 5, article 063, 32 pages, 2009.

[61] K. Lin and S. Yang, "Quantum tunnelling in charged black holes beyond the semi-classical approximation," Europhysics Letters, vol. 86, no. 2, Article ID 20006, 2009.

[62] K. Lin and S. Z. Yang, "Fermion tunnels of higher-dimensional anti-de Sitter Schwarzschild black hole and its corrected entropy," Physics Letters B, vol. 680, no. 5, pp. 506-509, 2009.

[63] S. W. Hawking, "Zeta function regularization of path integrals in curved spacetime," Communications in Mathematical Physics, vol. 55, no. 2, pp. 133-148, 1977.

[64] R. K. Kaul and P. Majumdar, "Logarithmic correction to the Bekenstein-Hawking entropy," Physical Review Letters, vol. 84, article 5255, 2000.

[65] D. V. Fursaev, “Temperature and entropy of a quantum black hole and conformal anomaly," Physical Review D, vol. 51, Article ID R5352, 1995. 
[66] S. Mukherjee and S. S. Pal, "Logarithmic corrections to black hole entropy and ADS/CFT correspondence," Journal of High Energy Physics, vol. 2002, no. 5, article 026, 2002.

[67] V. P. Frolov, W. Israel, and S. N. Solodukhin, "One-loop quantum corrections to the thermodynamics of charged black holes," Physical Review D, vol. 54, no. 4, pp. 2732-2745, 1996.

[68] M. R. Setare, "Logarithmic correction to the Cardy-Verlinde formula in topological Reissner-Nordström de Sitter space," Physics Letters B, vol. 573, pp. 173-180, 2003.

[69] M. R. Setare, "Logarithmic correction to the Brane equationin topological Reissner-Nordström de Sitter space," The European Physical Journal C-Particles and Fields, vol. 38, no. 3, pp. 389394, 2004.

[70] S. Das, P. Majumdar, and R. K. Bhaduri, "General logarithmic corrections to black-hole entropy," Classical and Quantum Gravity, vol. 19, no. 9, pp. 2355-2367, 2002. 

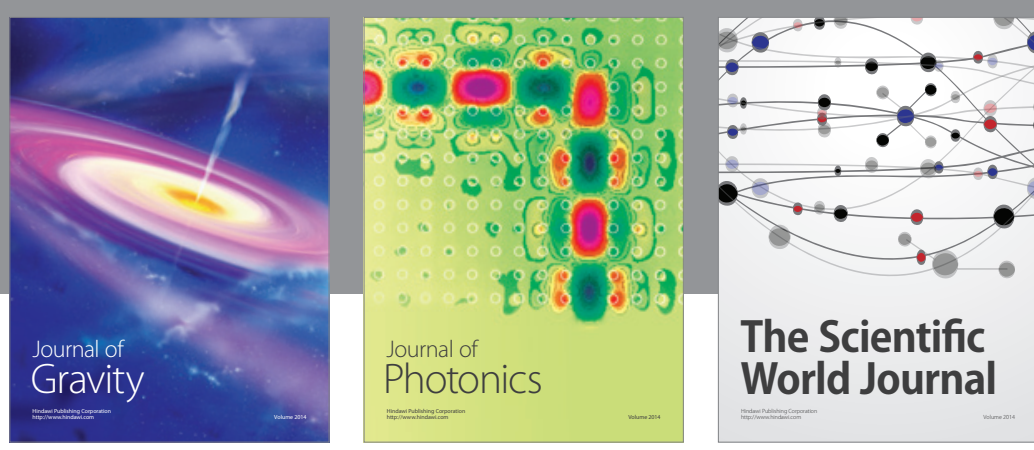

The Scientific World Journal
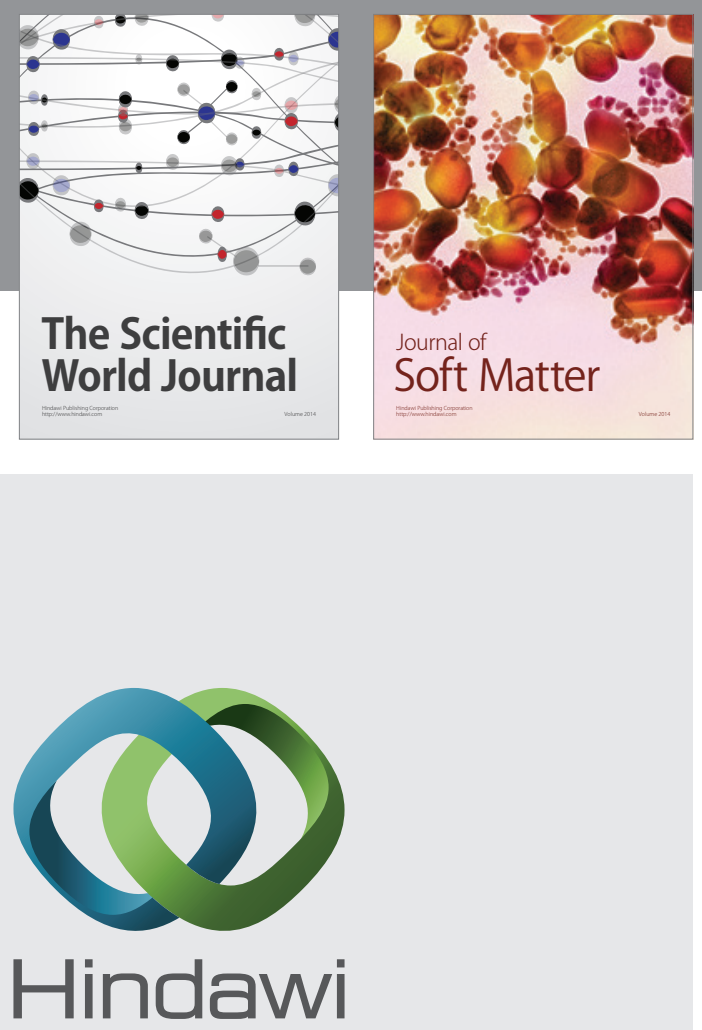

Submit your manuscripts at

http://www.hindawi.com

nternational Journal of

Statistical Mechanics
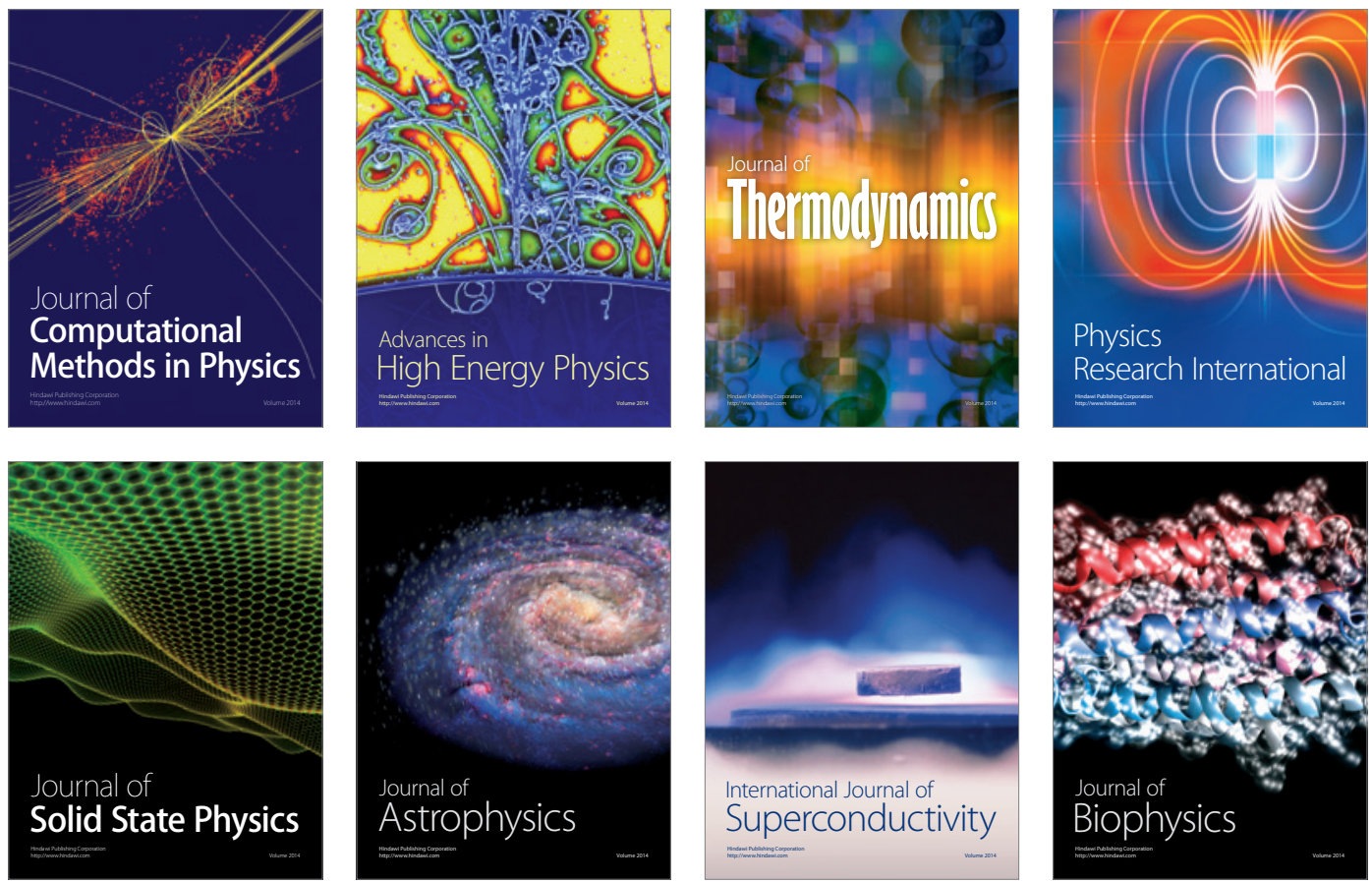
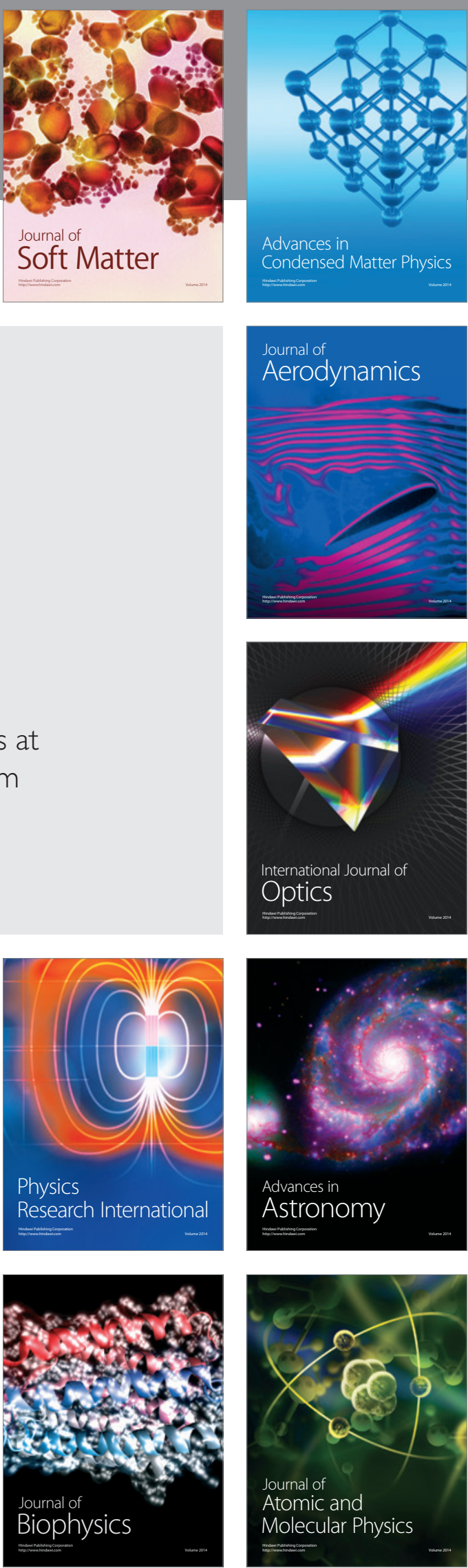